\title{
Feature Extraction Capability of Some Discrete Transforms
}

\author{
Ja-Ling Wu and Wei-Jou Duh \\ Department of Computer Science and Information Engineering, \\ National Taiwan University, 10764, Taipei, Taiwan, R.O.C.
}

\begin{abstract}
Feature extraction is a fundamental operation of classification and pattern recognition. There are various strategies for one- and multi-dimensional feature extraction. The transform domain features are very effective when the patterns are characterized by their spectral properties. A wellknown successful example is the speech recognition. In this paper the feature extraction capability of discrete cosine transform (DCT), Walsh-Hadamard transform (WHT), discrete Hartley transform (DHT) and their sign transformations are investigated and compared for the recognition of two dimensional binary patterns. It is shown, in this paper, that the noise immunity of the transform based feature extraction is rather promising.
\end{abstract}

\section{Selection of Transforms}

The appliance of unitary transforms to feature extraction had been discussed in $[1,2]$. Since the characteristics of speech signals are represented by their spectral properties, the adoption of unitary transforms for speech recognition is very intuitive and effective [5].

There are several ways to extract features of 2D patterns, such as amplitude features, histogram features, transform domain features and shape features. Some comparisons of various properties among the discrete Fourier transform (DFT), the Karhunen-Loève trans form (KLT), the WHT and the Haar transform (HT) were given in [1]. It is well known that KLT is the optimum choice for signal decorrelation in the sense of mean square error and the DCT performs closest to the KLT for highly correlated inputs. Hence, the feature extraction capability of the DCT is interesting.
The DCT was first proposed in 1974 [3] and was proven to be of closest performance to the KLT for Markov-I signal class [4]. Thus it is natural to use DCT for feature extraction, classification and pattern recognition. The previous works [1] shown that the DFT performs better than other unitary transforms in pattern classification applications. Since the transform kernel of DHT is very similar to that of DFT, the performance of the DHT is believed to be comparable to that of the DFT. Recently, Ersoy had proposed a new two - stage representation of the DFT [5]. In his work the computation of the DFT was decomposed into pre- and post processing stages by using Möbius inversion formula [6].

The pre-processing stage of the two-stage DFT consists of $\pm 1, \pm j$ and their combinations; the postprocessing stage consists of several independent convolvers or correlators of smaller sizes. The two-stage representation of the DFT is not only a fast algorithm but also provides a new tool for feature extraction. The simple pre-processing stage can be treated as a new transform called discrete rectangular wave transform (DRWT). Ersoy et al. have used the DRWT in speech recognition [7] and image recognition $[8,9]$ and the results are remarkable.

From the derivation of the two-stage DFT [5], the sine and cosine function can be represented in numbertheoretic bases. It is clear from [5] that the DRWT is, in fact, the sign transformation of the DFT. Some similar works of the two-stage representations of the DHT [10] and DCT [11] were also given. Following the feature extraction concepts of the DRWT $[7,8,9]$, the sign transformations of the DHT and DCT are also taken into consideration in the pattern recognition applications. Therefore, the feature extraction capability of five different discrete transforms, including the WHT, DHT, DCT, DHT-sign and DCT-sign, are investigated in detail. 


\section{Experiment Results}

Five different discrete transforms are considered in the experiment and two criterions are used for performance measurement, that is the absolute norm and the mean square error. Two sets of experiments are conducted, including standard numberical character set and similar character set. The standard numerical characters, say $\{0,1,2,3,4,5,6,7,8,9\}$ represented as $8 \times 8$ binary matrices, are used as the reference patterns of the experiments; and the similar character set is chosen as $\{0, D, Q\}$, each represented by a $16 \times 16$ binary matrix. The experiment procedures are as follows:

1. Determine the dimensionality of features in transform domain.

2. Transform the reference patterns and store the obtained features with pre-determined dimensionalities.

3. Generate three sets of test patterns from reference patterns with bit error rate $3 \%, 5 \%$ and $10 \%$, respectively.

4. Transform these noisy test patterns.

5. Calculate the absolute norm and the mean square error between the test and the reference patterns using the pre-determined feature dimensionalities.

6. Classify the test patterns to the corresponding minimum distance pattern.

\subsection{Experiments of numerical patterns}

Three sets of pattern recognition experiments were conducted, including bit error rate $3 \%, 5 \%$ and $10 \%$, respectively. With the same bit error rate, 200 test patterns corrupted by noise are generated and five different discrete transforms are carried out on the same set of test patterns using both absolute norm and mean square error criterions. The confusion matrices [7] for the WHT, DHT, DCT, DHT-sign and DCT-sign transforms are listed in table $1,2,3,4,5$, respectively. In these tabulated results, reference patterns are $8 \times 8$ binary characters, from 0 to 9 . Bit error rate is $5 \%$ and feature dimensionality is 8 . The experiment is repeated 200 times and the confusion matrices using mean square error criterion are listed.

From the previous experimental results, the recognition process based on absolute norm criterion always performs worse than that of the mean square error one.
In general, the orthogonal transforms are more sensitive to input patterns. That is, some patterns are easy to recognize and the other patterns are more confusable using the WHT, DHT and DCT; the performances of DCT-sign and DHT-sign are not so sensitive to input patterns but the correction rate are a little lower than the formers. In this experiment, the DHT and DHT-sign perform better than WHT; DCT-sign transform performs close to the WHT and the performance of the DCT is adequate compared to other transforms.

\subsection{Experiments of Similar Patterns}

The classification capability of similar patterns are of interest to most of the pattern recognition peoples. In this paper, the similar pattern set is chosen to be $\{O, D$, $Q\}$ as shown in figure.1. The experiments are carried out following the same strategies as in section 2.1. The results are shown in table $6,7,8,9$ and 10 .

\section{Discussions and Conclusions}

The use of discrete transforms for feature extraction and pattern recognition were common. The DFT achieves pretty good performance in the previous works [1]. Since the DRWT, the sign transformation of the DFT, can also be applied to this field and performs even better, the use of discrete Hartley, cosine, Hartley-sign and DCTsign transforms in the area of pattern recognition are of interest.

Since the transform kernels of the DFT and DHT are similar, the feature extraction capability of the DFT and DHT is deemed to be similar; so does DRWT and DHT-sign transform. The performances of the DCT and DCT-sign transform are close to WHT for normal numerical character set; the performance of noisy pattern recognition of the DCT is excellent for similar patterns. It is observed that the non-unitary sign transformations performs adequate and are not sensitive to input character sets. Thus, these sign transformations are suitable for general classification applications. Furthermore, from the experiments conducted in this paper, one can analysis the degree of noise immunity of various transforms for transform domain based pattern recognition. 


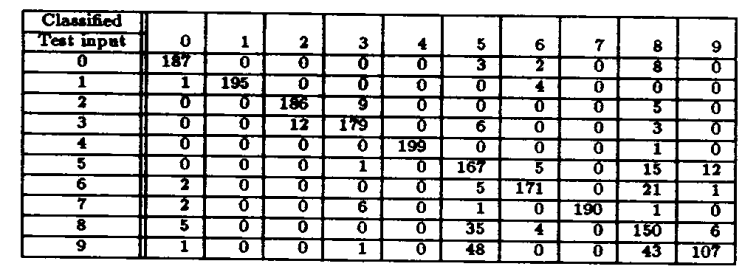

Table 1: The confusion matrix of the WHT

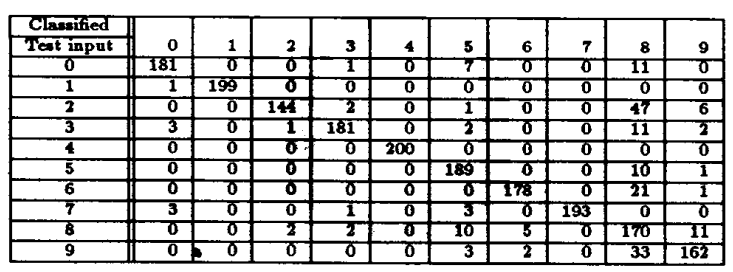

Table 2: The confusion matrix of the DHT

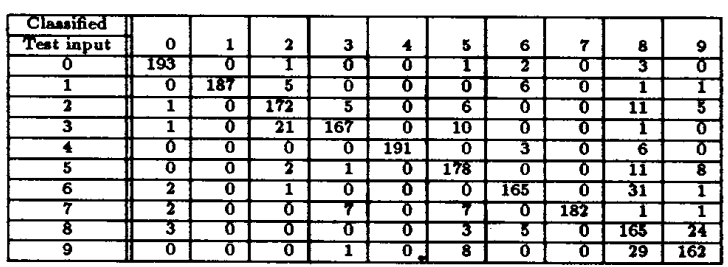

Table 3: The confusion matrix of the DCT

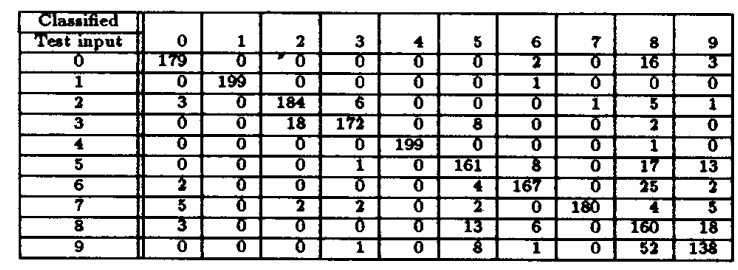

Table 4: The confusion matrix of the DHT-sign transformation

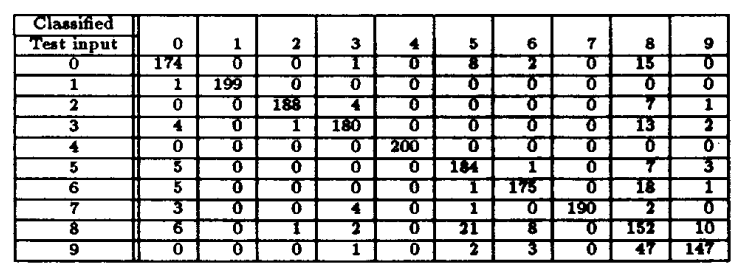

Table 5: The confusion matrix of the DCT-sign transformation

\begin{tabular}{|c||r|r|r|}
\hline Classified & & & \\
\cline { 1 - 3 } Test input & $\mathrm{O}$ & $\mathrm{D}$ & $\mathrm{Q}$ \\
\hline O & 4 & $\mathbf{0}$ & 6 \\
\hline D & 0 & 10 & 0 \\
\hline Q & 0 & 0 & 10 \\
\hline
\end{tabular}

Table 6: The confusion matrix of the WHT

\begin{tabular}{|c||r|r|r|}
\hline Classified & & & \\
\cline { 1 - 3 } Test input & O & D & Q \\
\hline O & 1 & 1 & 8 \\
\hline D & 0 & 10 & 0 \\
\hline Q & 0 & 0 & 10 \\
\hline
\end{tabular}

Table 7: The confusion matrix of the DHT

\begin{tabular}{|c||r|r|r|}
\hline Classified & & & \\
\cline { 1 - 3 } Test input & O & D & Q \\
\hline O & 7 & 1 & 2 \\
\hline D & 0 & 8 & 2 \\
\hline Q & 0 & 0 & 10 \\
\hline
\end{tabular}

Table 8: The confusion matrix of the DCT

\begin{tabular}{|c||c|c|c|}
\hline Classified & & & \\
\cline { 1 - 3 } Test input & O & D & Q \\
\hline O & 3 & 0 & 7 \\
\hline D & 0 & 9 & 1 \\
\hline Q & 0 & 1 & 9 \\
\hline
\end{tabular}

Table 9: The confusion matrix of the DHT-sign transformation 


\begin{tabular}{|c||r|r|r|}
\hline Classified & & & \\
\cline { 1 - 1 } Test input & O & D & Q \\
\hline O & 4 & 0 & 6 \\
\hline D & 0 & 10 & 0 \\
\hline Q & 0 & 0 & 10 \\
\hline
\end{tabular}

Table 10: The confusion matrix of the DCT-sign transformation

The error rate is $10 \%$ in this similar character recognition experiment. The feature dimensionality is 4 .

\section{References}

[1] N. Ahmed and K.R. Rao, Orthogonal Transforms for Digital Signal Processing. Springer-Verlag, NY, 1975.

[2] Anil K. Jain, Fundamentals of Digital Image Processing. Prentice Hall International co., 1989.

[3] N. Ahmed, T. Natarajan and K.R. Rao, "Discrete Cosine Transform," IEEE Trans. Computers, Vol.C-23, pp.90-93, Jan. 1974.

[4] Massih Hamidi and Judea Pearl, "Comparison of the Cosine and Fourier Transform of Markov-I Signals," IEEE Trans. Acoustic, Speech, and Signal Processing, pp428-429, Oct. 1976.

[5] Okan Ersoy, "A Two-Stage Representation of DFT and Its Applications," IEEE Trans. Acoustic, Speech and Signal Proçessing, Vol.ASSP-35, pp.825-831, June 1987.

[6] M.R. Schroeder, .Number Theory in Science and Communication. Springer-Verlag, 1983.

[7] D.Y. Kim, B.J. Stanton, O.K. Ersoy and L.H. Jamleson, "Speech Recognition with the Discrete Rectangular Wave Transform," ICASSP, pp.619-622, 1988.

[8] O.K. Ersoy and D.Y. Kim, "Image Recognition with the Discrete Rectangular Wave Transform," J.Opt.Soc.Am.A,'Vol.5, No.1, pp.5-18, Jan. 1988.

[9] D.Y. Kim and O.K. Ersoy, "Image Recognition with the Discrete Rectangular Wave Transform II," J.Opt.Soc.Am.A, Vol.6, No.6, pp.835-843, June 1989.

[10] Ja-Ling Wu and C.Y. Hsu, "Comments on: A Two-Stage Representation of DFT and Its Applications," IEEE Trans. ASSP, Vol.ASSP-36, p.1687, Oct. 1988.
[11] Ja-Ling Wu, Shyh-Huei Hsu and Wei-Jou Duh, "A Two Stage Convolver Based DCT and IDCT Algorithms," Submitted to IEE Proceedings.
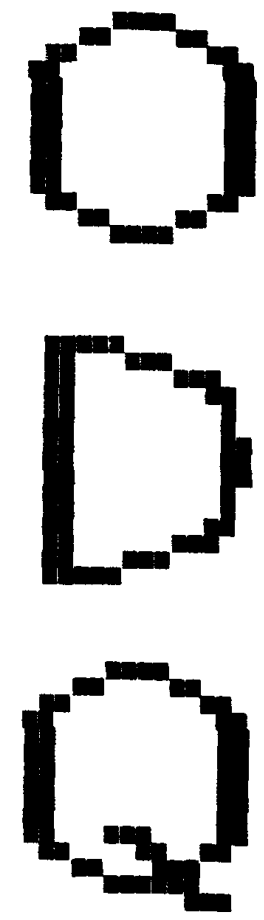

Figure 1: The similar character set 